\title{
The Electronically Activated Recorder (EAR): A device for sampling naturalistic daily activities and conversations
}

\author{
MATTHIAS R. MEHL and JAMES W. PENNEBAKER \\ University of Texas, Austin, Texas \\ D. MICHAEL CROW \\ Southern Methodist University, Dallas, Texas \\ JAMES DABBS \\ TGA Technologies, Inc., Norcross, Georgia \\ and \\ JOHN H. PRICE \\ Tipware.com, Austin, Texas
}

\begin{abstract}
A recording device called the Electronically Activated Recorder (EAR) is described. The EAR taperecords for 30 sec once every 12 min for 2-4 days. It is lightweight and portable, and it can be worn comfortably by participants in their natural environment. The acoustic data samples provide a nonobtrusive record of the language used and settings entered by the participant. Preliminary psychometric findings suggest that the EAR data accurately reflect individuals' natural social, linguistic, and psychological lives. The data presented in this article were collected with a first-generationEAR systembased on analog tape recording technology, but a second generation digital EAR is now available.
\end{abstract}

Across the social sciences, researchers often rely on selfreports to assess individuals' social and psychologicalstates. Although this subjective approach is certainly appropriate for some scientific questions, concerns have arisen as to whether people's actual behavior in real life situations can be captured by retrospective self-reports (for a thorough review, see Stone, Turkkan, Bachrach, Jobe, Kurtzman, and Cain, 1999). Studies of autobiographical memory show that people are usually not very accurate in recalling past events that have occurred years, weeks, days, or even just hours ago. Several factors bias remembering at all stages of human information processing (Tourangeau, 1999), including the salience of an event (Eisenhower, Mathiowetz, \& Morganstein, 1991), a person's implicit theories of stability and change (Ross, 1989), the evaluation of one's current situation (Bradburn, Rips, \& Shevell, 1987), and the emo-

We are indebted to Geetha Desikan, Kim Fisher, Beth Henary, Allison McClure, John McFarland, Kristy Orr, Nathalie Shook, Monica Sidarous, Mary-Beth Sylvester, and Sara Webb for their help in collecting the data and transcribing the audiotapes. Preparation of this paper was aided by Grant MH52391 to J.W.P. from the National Institutes of Health and by a scholarship to M.R.M. from the German National Scholarship Foundation. Correspondence should be addressed to J. W. Pennebaker, Department of Psychology, University of Texas, Austin, TX 78712 (email: pennebaker@psy.utexas.edu).

Note: J.H.P. has a financial interest in the production of the Electronically Activated Recorder (EAR). -Editor tional state during the encoding and retrieval of information (Blaney, 1986) - all of which are variables that in field studies typically fall outside of the experimenter's control.

Mihaly Csikszentmihalyiand his colleagues (e.g., Csikszentmihalyi \& Larson, 1987) were among the first to offer a solution to this problem. They developed the Experience Sampling Method (ESM), a procedure that requires participants to fill out questionnaires several times a day immediately after being beeped by a preprogrammed pager or wrist watch. Around the same time, William Brewer (1988) described a similar sampling technique aimed at assisting the systematic exploration of characteristics of autobiographical memory. Drawing on and extending these approaches, Arthur Stone and Saul Shiffman introduced Ecological Momentary Assessment (EMA), a tool designed for collecting psychological and even physiological moment-by-moment data in real-world settings, using recent computer technology such as palm-top computers to prompt participants (Stone \& Shiffman, 1994). These "online" self-reports bypass many of the problems of original techniques such as ESM, wherein participants could complete or alter their self-reports several hours after being beeped (Shiffman, 1999; Stone et al., 1998).

In this article, we introduce the ElectronicallyActivated Recorder (EAR), a new technology for obtaining behavioral data in field research, which captures ongoing behavior without relying on self-reports. Participants wear a small microcassette tape or digital voice recorder, which period- 
ically records brief intervals of their ambient sounds. The sound samples provide researchers with unobtrusive, immediate, and non-self-report information about a person's ongoing behaviors and immediate social milieu over the course of the day. From the recordings, real-world conversations can be transcribed, and assorted social and environmental information related to the participants can be reliably coded. Data from the EAR should be construed not as a substitute for self-reports, but rather as a complement to traditional assessment tools in the behavioral sciences.

First, we provide a technical description of the components of the EAR. Next, all the information that one needs in order to apply the system in field research is given. We then focus on specific EAR data analysis strategies. Finally, we address potential problems and limitations of this method.

\section{THE EAR RECORDING SYSTEM}

The EAR comprises a microcassette tape recorder (e.g., OPTIMUS Micro-32), an external microphone (e.g., OPTIMUS Omnidirectional Tie Clip Microphone), a controller computer chip, and an external battery pack. ${ }^{1}$ The EAR's microcassette recorder is operated by a custom controller board. The controller board is made up of a Microchip PIC16C62A microcontroller chip, a quartz crystal oscillator, and an analog circuitry capable of switching power to the microcassette recorder.

Every $12 \mathrm{~min}$, firmware programmed into the microcontroller chip switches the recorder on for a 30-sec sample. At the onset of each recording sample, a 3 - to 5-sec period is required for the recording volume to reach normal levels. This delay, experienced while internal recorder components approach their quiescent operating state, leaves about $25 \mathrm{sec}$ of useful data. In order to clearly separate recording samples for coding purposes, the controller injects a standard tone onto the tape between samples.

\section{APPLYING THE EAR IN FIELD RESEARCH}

In a pilot study, the EAR was tested on 52 introductory psychology students. The participants were asked to wear the EAR twice for 2 days separated by 4 weeks. This article focuses only on methodologicalconsiderationsfor using and assessing the EAR. The results of the study will be published elsewhere.

\section{Setting Up the System}

The EAR was packed into a small, durable, padded camera case, which could be worn around the waist or over the shoulder like a purse. Figure 1 shows how the system looks when worn by a participant.

All parts of the monitor except the stop, record, and play buttons - which were to be used by the participantswere taped over to prevent inadvertent misuse. The external microphone, clipped to the top of the bag, pointed toward the head of the person. Participants were advised to be sure that their clothes did not cover the microphone.
It is important to note that these guidelines for appropriate use are the result of several rounds of technical improvements. As part of the EAR development and optimization process, research assistants as well as the main investigators of the project engaged in extensive field pretesting, wearing the device themselves several times for several days.

The authors' own experiences correspond to the reports of the research assistants that it was not possible to hear, feel, or see the monitor (packed in the padded case, as described above) as it starts and stops recording, even if one explicitly intends to do so. When asked about this in the debriefing session, the participants of the pilot study also confirmed that they were unable to sense when the EAR was on or off.

\section{Introducing the EAR to the Participants}

When the participants arrived for the first session, they were informed that the study was designed to assess people's daily lives and the environments in which they naturally found themselves. As part of the research, the participants learned that we were interested in discovering the various sounds that surrounded them as they went about their daily activities. It was explained that the various sounds and other acoustic data that were captured on the tape recordings would be evaluated by researchers. The

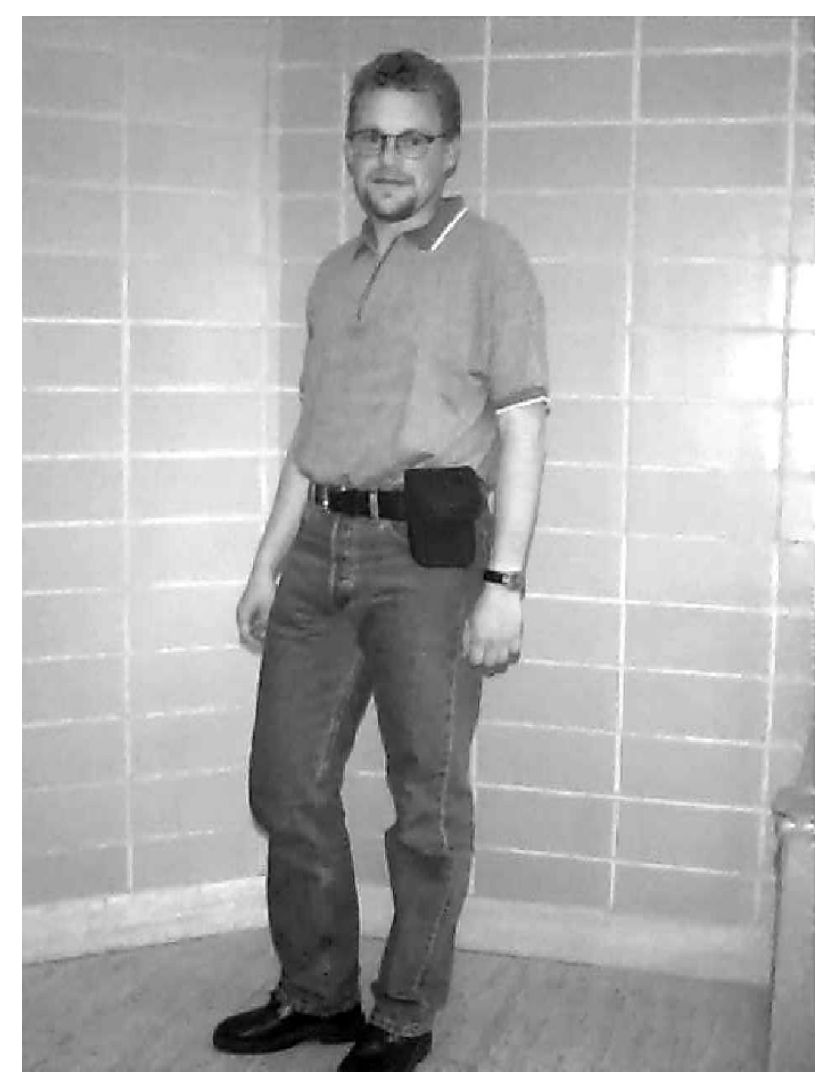

Figure 1. Illustration of the Electronically Activated Recorder (EAR), here worn on a belt. 
participants were then shown the device and were told that the monitor switched on at random intervals throughout the day- - even though, in fact, it followed a specified, periodic recording pattern.

Virtually all participants readily agreed to wear the EAR. Some common concerns included those of being impeded in daily activities by wearing a rather unwieldy device, being exposed to gazes of other people who happened to see the microphone, having to wear the EAR overnight, and having embarrassing conversations recorded on the tape. We addressed these concerns in the following manner.

First, the participants were shown the EAR bags and given instructions on how to carry them. It was mentioned that when it might be too uncomfortable to wear (e.g., while one was sitting in a chair), they could also put it beside them on the table. Next, a brief instruction manual was handed out. It contained all relevant information about appropriate handling, specified times when they were to wear the monitor, and the phone number of the experimenter's office for help in dealing with any technical problems. The participants were then advised about how to deal with questions or comments from other people. Basically, they were encouraged to mention that they were part of a scientific study and that all the recordings would be kept confidential.

The issues of security and privacy were discussed in detail with all participants. It was emphasized that the recorder only captured 30 -sec blocks at random intervals rather than extended conversations. More importantly, at the end of the study before the experimenters ever listened to the tapes, all participants were given the option of reviewing their recordings and erasing anything they considered objectionable. After being given all this information regarding our confidentiality policies, only 2 out of 54 students were not willing to sign the experiment's consent form, with only 1 explicitly stating that the EAR was the reason.

The participants initially read identifying information (i.e., date, subject number, and monitoring session) onto the tape. This served as a voice sample so that the transcribers could distinguish between the voice of the person wearing the EAR and that of his or her conversation partners. The students were then instructed to wear the monitor for 2 days, switching the recorder off only at night. It was emphasized that obtaining as many hours of recording as possible was critical, but the device should be removed when its functioning was jeopardized (i.e., it might be exposed to water, or the participant might be engaged in strenuous exercise). Finally, the system was activated by the experimenter.

\section{Feedback}

When the participants returned to the lab to turn in the EAR (i.e. twice, separated by 4 weeks), they completed a questionnaire about their experiences with the system. All participants were asked how comfortable they felt wearing the EAR, how aware they themselves and other people were of the monitor, how much the system changed their and other people's behavior, and how much they talked about the EAR. In addition, they were allotted free space in which to make comments about the monitor. Table 1 lists the items with the respective means and standard deviations for the two monitoring periods.

The descriptive statistics show that the participants felt fairly comfortable wearing the EAR. Note that all questionnaire items were based on a 5-point unipolar scale, with 1 indicating not at all and 5 indicating a great deal. As can be seen in the table, participants were less aware of the EAR, felt that other people were less influenced by it, and talked less about it during the second 2-day monitoring period than during the first.

The students' comments painted the following picture: During the first few hours, they felt slightly impeded by the bag and tended to be more conscious of what they were doing and saying. Any uneasiness caused by the EAR usually subsided after a couple of hours, resulting in the participants' rarely noticing it toward the end of the 1st day. Some even forgot to turn it off at night - showing that they were not overly conscious of being recorded. Others who saw the device were sometimes bothered by it at first, wondering about the confidentiality of the recordings. Participants' male friends often enjoyed performing in front of the recorder, hoping to be captured on tape. There were almost no spontaneous comments on the questionnaire after the second 2 days of monitoring.

At the final session, the participants were asked whether or not they ever voluntarily turned the recorder off. Only

Table 1

Item-Means of Self-Reported Experiences with the EAR During the First and Second Monitoring Periods

\begin{tabular}{|c|c|c|c|c|c|}
\hline \multirow[b]{2}{*}{ Item } & \multicolumn{2}{|c|}{ 1st Two Days } & \multicolumn{3}{|c|}{ 2nd Two Days } \\
\hline & $M$ & $S D$ & $M$ & $S D$ & $p<$ \\
\hline \multicolumn{6}{|l|}{ To what degree } \\
\hline did you feel uncomfortable wearing the EAR? & 2.18 & .97 & 1.92 & .84 & \\
\hline were you generally aware of the EAR? & 2.94 & .99 & 2.43 & .96 & .01 \\
\hline did the EAR change your actual behavior? & 1.49 & .67 & 1.39 & .63 & \\
\hline did the microphone influence your way of talking? & 1.31 & .71 & 1.20 & .40 & \\
\hline did other people recognize the EAR? & 2.80 & 1.15 & 2.55 & .99 & \\
\hline did the EAR influence other people's behavior? & 2.17 & 1.20 & 1.77 & 1.00 & .01 \\
\hline did you talk about the EAR to other people? & 3.20 & .98 & 2.20 & .80 & .001 \\
\hline
\end{tabular}

Note- $N=52$; significance levels are two-tailed probabilities based on a paired $t$ test; ratings range from 1 , indicating not at all, to 5, indicating a great deal. 
2 of the 52 participants said that they turned it off; in both cases this happened during discussions with their significant others. Only 1 of them took the opportunity to listen to parts of his tapes, and did not erase anything.

Interestingly, participants showed a remarkably high degree of commitment, sometimes even employing creative means to capture problematic situations. Some students reported putting the EAR next to them when running on a treadmill in the gym; one male participant placed it in the corner of the studio during his karate lesson. Another participant who also worked as a waiter wore the EAR underneath his apron. Overall, the fact that the participants were willing to cooperate with the experimenter indicated their comfort with and interest in the system.

\section{DATA ANALYSIS STRATEGIES}

Data from the EAR provide a rich source of information both about the settings of the participants as well as the ways they interact with others. To date, we have adopted two methods of coding and analyzing EAR data: text analyses of the words used by the participant and those in the participants' environment, and judges' ratings of situational factors. As can be seen in Table 2, both of these strategies are typically done concurrently by a transcriber who first transcribes the 30 -sec time block and then rates each of several situational and behavioral categories.

\section{Transcriptions of Language}

For each 30-sec block, transcribers break all language samples as originating from one of three sources: the subject $(\mathrm{S})$, another live person $(\mathrm{O})$ talking with or in the immediate proximity of the person, or language from an impersonal source such as the media (M) or a lecturer. The media category includes words from songs and commercials on the radio as well as words from a play that a participant may be attending.

Data from the transcripts are typically analyzed in two ways. The first involves sorting the language sample by speaker (, , O, or $\mathrm{M})$ and then submitting the text to a computer text analysis program, such as Linguistic Inquiry and Word Count, or LIWC (Pennebaker, Francis, \& Booth, 2001). Such a program calculates the percentage of words within each text sample that can be classified along over 70 linguistic dimensions (e.g., positive or negative emotion, self-references, etc.). Using this strategy, for example, we can compare (1) the percentage of positive emotion words that the participant uses in the weeks before an intervention such as the emotional writing paradigm (for details see Pennebaker, 1997) or any other psychological or behavioral treatment with (2) the percentage during the recording period in the weeks after the intervention.

\section{Psychometric Properties of Judges' Ratings of Context}

Each 30-sec time block is also coded along multiple dimensions by the transcriber to pinpoint the setting and associated behaviors that are ongoing during the time block.
As is apparent in Table 2, several categories within three broad dimensions are coded: Conversation, Activity, and Environment. Conversational dimensions include whether or not the participant is talking, is on the phone, or laughs at least once during the 30 -sec period; the number of other people talking (according to the captured voices); and the number of other persons present (guessed by the covered background noises). The Activity category refers to ongoing behaviors or tasks in which the person is involved: listening to the radio or watching television, typing on a computer (typing noise), reading/studying (applied when the judges were listening to silence interrupted by the noise of turning pages or the writing of a pen), working (e.g., as cashier at a supermarket, waiter in a coffee shop), eating (including the preparation of meals), listening to a lecture, or participating in an activity deemed an amusement (e.g., playing video games, watching a movie). The Environment category attempts to place the person in a particular location: at the participant's apartment/home, outdoors, in transit (e.g., walking, in a car), in a restaurant, or in some other public location. All of these categories are rated in a binary fashion (presence vs. absence), except for the numbers of people talking or present, which are simply raw counts.

Six research assistants underwent two phases of training and calibration to get expertise in applying the coding system to the acoustic data. In the first phase, the coding system was explained and a demonstration tape was rated by all of them simultaneously under supervision of the first author. This gave us the opportunity to specify the meaning of every category and clarify potential ambiguities. In the second phase, each assistant individually transcribed and coded a calibration tape ( 88 intervals). Reliability analyses run with the calibration data confirmed high interrater agreements for most categories ("Judges' Reliability" in Table 3; Cronbach's $\alpha \geq .85$ in 14 out of 18 categories) but also pointed to areas where the current system needs to be refined for future use (e.g., the categories "Reading," "Eating," "Amusement," and "Restaurant").

As described above, the participants were asked to wear the EAR twice for 2 entire days separated by 4 weeks. To give an impression of the base rates of the captured behaviors and situational aspects, Table 3 shows the proportion of sampled intervals (mean and standard deviation), for each monitoring period, in which behavior in each of the 18 categories was recorded. Note, for example, that a mean value of .273 for the variable "subject talking" during the first monitoring period indicates that the average participant was talking in $27.3 \%$ of the intervals. Also, the average participant during the first monitoring period was on the phone $3.8 \%$ of the recorded time, was laughing in $5.9 \%$ of the cases, and spent about half of his day $(50.7 \%)$ inside an apartment. During the second monitoring period, the rate for "subject talking" slightly decreased to $24.8 \%$, participants were on the phone $3.4 \%$ of their time awake, laughing in $4.9 \%$ of the cases, and inside an apartment in $56 \%$ of the intervals. These descriptive data are mentioned to demonstrate more vividly how the EAR offers insight into the participants' daily lives. Finally, Table 3 also depicts 


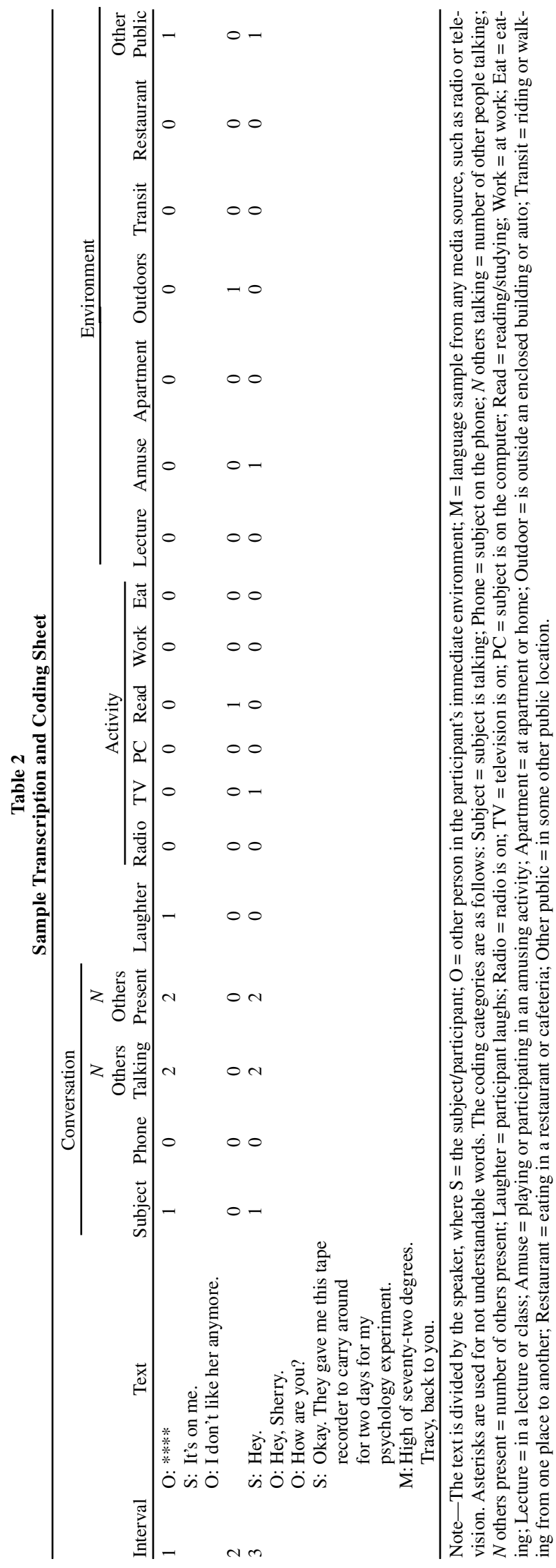

1-month stabilities of all 18 EAR categories. Test-retest correlations range from .15 to .76 , with an average of $r(50)=.53$.

\section{Psychometric Properties of Natural Language Captured by the EAR}

From the oral transcripts, we were able to extract linguistic categories produced by the text analysis program LIWC (Pennebaker et al., 2001). Table 4 shows simple correlation coefficients between the major linguistic variables of the first and the second monitoring periods. The reliability of the linguistic variables over the 4 -week period range from $r(50)=.09$ (past tense words) to $r(50)=.60$ (filler words), with an arithmetic mean of $r(50)=.36$. Although these data should be considered as preliminary, they point to the remarkable stability of the ways in which individuals talk across time and setting. They also underscore the importance of considering linguistic styles as a way of thinking about individual differences (Pennebaker \& King, 1999).

Taken together, the psychometric properties of the EAR are promising. Equally intriguing are potential findings that will link the EAR data with information obtained from self-reports. Indeed, this technology will also allow the researcher to monitor subtle traces of behavior that people do not readily have access to (e.g., the amount of time spent talking or laughing), as well as natural samples of their daily language use (e.g., distributions of word frequencies). This allows one to explore the impact of yet unstudied behavioral characteristics of a person and his or her linguistic style on the wide array of psychologicalphenomena.

\section{LIMITATIONS OF THE EAR}

The EAR adds new ways of looking at social psychological phenomena. In capturing people's conversations, behaviors, and settings directly from their immediate acoustical environments, a researcher can bypass some of the major criticisms of traditional retrospective field research and collect data that are not accessible via self-report. However, implementing the EAR system raises a number of practical and ethical issues.

At the most basic level, the collection of data using the EAR is time-intensive for both the participant and the researcher. At the outset, the nature of the EAR system must be explained to the participants in great detail. Participants are asked to wear the EAR during their waking hours for up to 4 days and to avoid dropping it, getting it wet, or - most important-losing it. In other words, prospective participants must allow the EAR to enter into all parts of their lives.

From the researchers' perspective, collecting, transcribing, and analyzing the EAR data is a massive undertaking. For each participant, there is a large number of separate 30 -sec recordings. The recording quality of some of the taped segments can be bad, because of ambient noises, low speaking volume, or quirks in the microphone orientation. It is not uncommon for participants to speak languages that transcribers do not understand. In addition, it 
Table 3

Descriptive Statistics and Psychometric Properties of the Judges' Context Ratings: Mean Occurrences and Standard Deviations for Both Monitoring Periods, 1-Month-Stabilities, and Reliability Coefficients for all 18 Categories of the EAR Coding System

\begin{tabular}{|c|c|c|c|c|c|c|}
\hline \multirow[b]{2}{*}{ Variable } & \multicolumn{2}{|c|}{ 1st Monitoring Period } & \multicolumn{2}{|c|}{ 2nd Monitoring Period } & \multirow{2}{*}{$\begin{array}{l}\text { 1-Month } \\
\text { Stability }\end{array}$} & \multirow{2}{*}{$\begin{array}{c}\text { Judges' } \\
\text { Reliability } \\
\end{array}$} \\
\hline & $M$ & $S D$ & $M$ & $S D$ & & \\
\hline Subject talking & 273 & .136 & .248 & .118 & .54 & .99 \\
\hline Talking on phone & .038 & .038 & .034 & .038 & .36 & 1.0 \\
\hline$N$ others talking & .376 & .237 & .306 & .183 & .52 & .96 \\
\hline$N$ others present & 444 & .317 & .359 & .227 & .65 & .93 \\
\hline Laughing & .059 & .043 & .049 & .039 & .53 & .86 \\
\hline Radio & 128 & .102 & .133 & .114 & .46 & 89 \\
\hline TV & .123 & .120 & .161 & .147 & .60 & .95 \\
\hline Computer & 077 & .092 & .081 & .081 & .30 & .96 \\
\hline Reading & .106 & .111 & .118 & .139 & .43 & .12 \\
\hline Working & .045 & .088 & .049 & .108 & .74 & .95 \\
\hline Eating & .035 & .039 & .018 & .021 & .15 & .64 \\
\hline Lecture & .111 & .076 & .108 & .081 & .34 & .98 \\
\hline Amusement & .117 & .163 & .145 & .189 & .67 & .71 \\
\hline Apartment & .507 & .190 & .562 & .234 & .46 & .97 \\
\hline Outdoor & .059 & .039 & .071 & .052 & .69 & .90 \\
\hline In transit & .045 & .046 & .035 & .042 & .76 & .85 \\
\hline Restaurant & .027 & .041 & .014 & .036 & .65 & - \\
\hline Other public places & .267 & .150 & .265 & .188 & .37 & .86 \\
\hline
\end{tabular}

Note $-N=52$; reliability coefficients are Cronbach's alphas based on six transcribers scoring 88 intervals; for the category "Restaurant," no reliability coefficient could be calculated because of lack of variance in the ratings.

is often difficult to determine what situation the participant may be in. Without a good sense of context, it can often be impossible for a transcriber to understand what the participant is saying or for the rater to evaluate features of the participant's environment.

Perhaps the thorniest issue surrounding the EAR concerns confidentiality. By definition, the participant is aware that his or her words are being recorded. However, others within range of the microphone may not know about the recorder. Strictly speaking, then, snippets of others' conversations may be recorded without their consent. In most states in the United States this is entirely legal. In a few (e.g., Linda Tripp's Maryland), permission of all people whose voices are being recorded must be obtained. Note that the legal and ethical questions are further clouded by the fact that typically the identities of nonparticipants' voices are not revealed, so that in theory, confidentiality and anonymity are maintained.

In our pilot study, only 1 of the original 54 participants refused to wear the EAR system after being informed how it worked. That participants were given the option of listening to the recordings and erasing them before turning them over to the researchers helped to allay potential anxieties about the system. However, some groups could be more sensitive about this technology than others (e.g., employees being asked to wear it at work or elderly people having more serious concerns about intrusion into their privacy).

The development of the EAR system is still in its infancy. Future studies must be undertaken to optimize technical aspects of the device and improve the efficiency of capturing data with the least amount of recording. Future research must also assess EAR data obtained in different populations and settings. Finally, the refinement of the processing of the raw data (transcribing, rating) requires additional conceptual and empirical work. Despite these challenges, we remain optimistic that the data provided by the EAR can yield new perspectives for studying people's natural social behavior and improving our understanding of basic social, cognitive, linguistic, and other psychological processes.

\section{RECENT DEVELOPMENTS OF THE EAR}

Since the first submission of this article our lab has been working on improving and refining the system. In terms of the underlying technology, a second generation recorder is now ready to be used in future field studies. The new EAR still records on repeating cycles of $30 \mathrm{sec}$ every $12 \mathrm{~min}$; its major improvement lies in a digital recording technique (Sony Memory Stick IC Recorder ICDMS1) instead of the analog microtape cassette recorder.

Table 4

4-Week Stability of the Major Linguistic Variables Obtained From a LIWC Analysis of the EAR Transcripts

\begin{tabular}{lc}
\hline Linguistic Variable & 4-Week Stability \\
\hline I, me, my & .19 \\
We, us, our & $.34^{*}$ \\
Prepositions & $.50^{* *}$ \\
Articles & $.36^{*}$ \\
Fillers (like, well) & $.60^{* *}$ \\
Past tense & .09 \\
Present tense & $.44^{* *}$ \\
Pos. emotion & .18 \\
Neg. emotion & $.49^{* *}$ \\
Causation words & $.34^{*}$ \\
\hline
\end{tabular}

Note- $N=52 . * p<.05 .{ }^{*} * p<.01$. 
The digital recorder can be equipped with up to $64 \mathrm{MB}$ of RAM, allowing up to 260 min of recording. As with the old system, a controller chip sends alternating "on" and "off" signals in the $12 \mathrm{~min} / 30 \mathrm{sec}$ pattern. Every sound sample is stored as a separate file onto the Memory Stick (in a Sony-specific file format that can be converted into an 8-bit or a 16-bit wav-file). Along with every 30 -sec recording, the recorder provides background information such as the date, time, and duration of the recording. It also creates a directory of all the stored messages that can be customized according to the user's needs (individually labeled and organized folders). Once the capacity of the Memory Stick is reached (over 4 days of continuous recordings), the recorded files can be easily downloaded onto the hard drive of a computer by using a Memory Stick adaptor (internal or external). The samples can then be listened to and edited with special voice editor software provided by Sony (for the Sony file format) or any other commercially available voice editor (for the wav-format).

Harnessing this new digital message technology for the EAR system brings with it several substantial practical advantages and methodologicalimprovements. With the new device being smaller, more light-weight (3.1 oz., including batteries and Memory Stick), and absolutely noiseless in its recording, the EAR is even less obtrusive for the environment and more comfortable for the participant. In addition, it is less shock sensitive, and it provides a better recording quality, markedly increasing the reliability of the system.

In addition to the changes in the recorder itself, we have also redesigned the microphone system in order to further ensure the privacy of nonparticipants. Specifically, we have now developed a lapel-like microphone that can be worn under the participant's clothing that will generally capture only the participant's voice - rather than all voices in proximity to the EAR wearer.

With this second generation technology, it is also very easy to launch a study matching the timed EAR information with any self-report, behavioral, or physiologicaldata collected in the field (e.g., journal entries, questionnaires, heart rate monitoring, salivary hormone levels). Finally, the opportunity to manage the data on the computer is not only helpful in terms of efficient data storage, it also makes it easier for the participants to listen to parts of their recording without spending time forwarding and rewinding the tape in an effort to locate the intervals of interest. This too is critical for adequately addressing the issues of privacy and data confidentiality, and it contributes to making the participants feel comfortable about wearing the EAR.

\section{AVAILABILITY}

The chip designed for the first-generation EAR with the necessary technical information for running it is available from James Dabbs. Researchers interested in using this system should contact him at TGA Technologies, Inc., Suite 140, 100 Pinnacle Way, Norcross, GA 30071 (e-mail: jdabbs@ tga.com).
The new 2nd generation EARs, including the Sony digital recorder and $64 \mathrm{~K}$ memory stick, are available for approximately $\$ 800$ each through the fifth author, John $\mathrm{H}$. Price. For information concerning sales or technical issues surrounding the EAR, please contact James W. Pennebaker at the University of Texas, Department of Psychology, Austin, TX 78712 (e-mail: pennebaker@ psy. utexas.edu).

Since the EAR is still in the initial stage of development, any suggestions on how to improve it are highly appreciated.

\section{REFERENCES}

Blaney, P. H. (1986). Affect and memory: A review. Psychological Bulletin, 99, 229-246.

Bradburn, N. M., Rips, L. J., \& Shevell, S. K. (1987). Answering autobiographical questions: The impact of memory and inference on surveys. Science, 236, 157-161.

BREWER, W. F. (1988). Memory for randomly sampled autobiographical events. In U. Neisser \& E. Winograd (Eds.), Remembering reconsidered: Ecological and traditional approaches to the study of memory (pp. 21-90). Cambridge: Cambridge University Press.

Csikszentminalyi, M., \& LARson, R. (1987). Validity and reliability of the experience-sampling method. Journal of Nervous \& Mental Disease, 175, 526-536.

Eisenhower, D., Mathiowetz, N. A., \& Morganstein, D. (1991). Recall error: Sources and bias reduction techniques. In P. Biemer, R. Groves, L. Lyberg, N. Mathiowetz, \& S. Sudman (Eds.), Measurement errors in surveys (pp. 127-144). New York: Wiley.

Pennebaker, J. W. (1997). Writing about emotional experiences as a therapeutic process. Psychological Science, 8, 162-166.

Pennebaker, J. W., Francis, M. E., \& Booth, R. J. (2001). Linguistic inquiry and word count: LIWC (2nd ed.) [Computer software]. Mahwah, NJ: Erlbaum.

PennebaKer, J. W., \& KInG, L. A. (1999). Linguistic styles: Language use as an individual difference. Journal of Personality \& Social Psychology, 77, 1296-1312.

Ross, M. (1989). Relation of implicit theories to the construction of personal histories. Psychological Review, 96, 341-357.

SHIFFMAN, S. (1999). Real-time self-report of momentary states in the natural environment: Computerized ecological momentary assessment. In A. A. Stone, J. S. Turkkan, C. A. Bachrach, J. B. Jobe, H. S. Kurtzman, \& V. S. Cain (Eds.), The science of self-report: Implications for research and practice (pp. 277-296). Mahwah, NJ: Erlbaum.

Stone, A. A., Schwartz, J. E., Neale, J. M., Shiffman, S., Marco, C. A., Hickcox, M., Paty, J., Porter, L. S., \& Cruise, L. J. (1998). A comparison of coping assessed by ecological momentary assessment and retrospective recall. Journal of Personality \& Social Psychology, 74, 1670-1680.

Stone, A. A., \& Shiffman, S. (1994). Ecological momentary assessment (EMA) in behavioral medicine. Annals of Behavioral Medicine, 16, 199-202.

Stone, A. A., Turkkan, J. S., Bachrach, C. A., Jobe, J. B., KurtzMAN, H. S., \& CAIN, V. S. (1999). The science of self-report: Implications for research and practice. Mahwah, NJ: Erlbaum.

Tourangeau, R. (1999). Remembering what happened: Memory errors and survey reports. In A. A. Stone, J. S. Turkkan, C. A. Bachrach, J. B. Jobe, H. S. Kurtzman, \& V. S. Cain (Eds.), The science of selfreport: Implications for research and practice (pp. 29-47). Mahwah, NJ: Erlbaum.

\section{NOTE}

1. The original EAR system-which is described in this section-was designed and tested between 1995 and 1999. For a brief description of the most recent version of the EAR, see the final section of the paper.

(Manuscript received February 10, 1999; revision accepted for publication March 21, 2001.) 